\title{
AdapterGame e suas Contribuições na Concepção de Jogos Educacionais
}

\author{
Cristina Paludo Santos, Vanio Rodrigo Reichel Bourscheidt
}

Universidade Regional Integrada do Alto Uruguai e das Missões (URI)

98.802-470 - Santo Ângelo - RS - Brasil

paludo@santoangelo.uri.br, vanior px@hotmail.com

\begin{abstract}
This paper presents a computational solution, referred to as AdapterGame, which provides resources to guide the design of games with adapted content. In the educational context, its use is related to the production of materials that serve as support for teaching practices, promoting subsidies for the provision of games that can be used in different disciplines and addressing diverse content. To evaluate the applicability of the proposed solution, educators have participated in the assessment process, allowing to identify strengths and important notes for improvement enhancement and extension of the proposed solution.
\end{abstract}

Resumo. Este artigo apresenta uma solução computacional, referenciada como AdapterGame, que provê recursos para direcionar a concepção de jogos com adaptação de conteúdo. Seu uso no contexto educacional está atrelado à produção de materiais de apoio às práticas pedagógicas, promovendo subsídios para a disponibilização de jogos que possam ser utilizados em diferentes disciplinas e que abordem diversos conteúdos. Como forma de avaliar a aplicabilidade da solução proposta, contou-se com o apoio de educadores que vislumbraram pontos positivos e fizeram apontamentos importantes para a melhoria e extensão da solução proposta.

\section{Introdução}

As tecnologias da computação evoluíram durante sua existência a ponto de abranger hoje a maioria dos campos de trabalho e estudo, desde controles de máquinas em fábricas até aplicações na medicina e na educação. Essa evolução tem propiciado a concepção de inúmeros recursos tecnológicos que visam enriquecer a semântica das aplicações computacionais e prover funcionalidades que aproximem mais o homem da máquina, de maneira transparente.

A necessidade dos usuários de que os sistemas sejam mais flexíveis, adaptáveis, interativos e fáceis de usar, apresenta-se como um desafio na área computacional e tem gerado várias pesquisas que propõem diferentes estratégias para o oferecimento de serviços mais adaptáveis às necessidades e intenções dos usuários. Dentre as várias áreas computacionais que estão sendo influenciadas pelas novas demandas de interação, destaca-se, neste trabalho, a área de jogos e, em particular, àqueles voltados à área educacional.

No contexto atual, os jogos têm sido considerados recursos didático-pedagógicos valiosos e com comprovadas contribuições no processo de ensino [Kishimoto 2010][Macedo 2009]. No entanto, possuem um processo de desenvolvimento padronizado, sem considerar a evolução da educação que cria um cenário onde as 
técnicas e métodos de ensino utilizados em uma determinada etapa se tornam defasados para a próxima, criando aplicações que dispõem de conteúdos estáticos, que uma vez utilizadas e assimilados seus conteúdos, perdem sua utilidade e as mesmas não poderão ser aplicadas novamente àquele aprendiz. Desta forma, surgem as seguintes questões:

(a) Como possibilitar a personalização e adaptação de conteúdo de forma dinâmica em jogos educacionais provendo a possibilidade do mesmo jogo ser utilizado para o ensino de diferentes conteúdos em distintas disciplinas?

(b) Como tratar individualmente as necessidades educacionais de cada aluno, aplicando atividades semelhantes no mesmo ambiente, para que cada um seja exposto ao conteúdo necessário para o seu desenvolvimento?

(c) Como desenvolver um software que tenha uma aplicação prolongada sem a diminuição dos seus benefícios, aumentando o aproveitamento da pesquisa realizada?

Nesta perspectiva, dois cenários devem ser considerados: (I) Jogos educacionais possuem conteúdo estático, não promovendo a dinamicidade, frequentemente necessária ao processo de ensino e, (II) Jogos educacionais não dispõem de flexibilidade para aplicação de conteúdos distintos a cada usuário, abordando suas necessidades específicas. Tais questões e cenários impulsionam a proposta do AdapterGame que permite a concepção de jogos com sensibilidade ao conteúdo, considerando as particularidades inerentes ao processo de ensino e aprendizagem. Tal modelo pretende promover a dinamicidade em jogos educacionais; incorporar recursos que possibilitem a avaliação do desempenho dos aprendizes e, possibilitar a inclusão de novos jogos utilizando o modelo proposto.

Uma descrição mais detalhada da proposta do AdapterGame é apresentada nas seções subsequentes, estruturadas da seguinte forma: a Seção 2 apresenta os princípios e teorias que norteiam o desenvolvimento do AdapterGame; na Seção 3, apresenta a estrutura inicial do sistema, bem como um esboço de uma das ferramentas disponibilizadas pelo sistema; por fim, a seção 4, apresenta as considerações finais.

\section{Principíos Norteadores do AdapterGame}

Em geral, jogos visam exclusivamente o entretenimento. Porém, com o desenvolvimento tecnológico aliado à abertura das escolas para novas práticas de ensino, os jogos educacionais computadorizados surgem como uma forma de auxiliar alunos e professores no processo de ensino e aprendizado [Hodgson 2010]. O uso de jogos educativos ou jogos sérios no ambiente educacional é uma tendência importante na área de pesquisa em educação baseada na informática, impulsionada pela capacidade dos jogos de evitarem vários problemas relacionados à frustração e à falta de motivação do aprendiz [Dillenburg 2011], tendo em vista que é comprovado o aumento da concentração, dos reflexos e da capacidade de tomada de decisões rápidas por jogadores [Morsi 2007].

Um dos objetivos dos jogos educacionais é prover meios para produção e construção do conhecimento pelo educando. Assim, os jogos educacionais podem se inserir em diversas atividades, as quais não estão voltadas apenas para o desenvolvimento de conteúdos específicos, mas como também de habilidades que enriquecerão a formação geral do educando, auxiliando-o a: ampliar sua linguagem e 
promover a comunicação de idéias; adquirir estratégias de resolução de problemas e de planejamento de ações; desenvolver a capacidade de fazer estimativas e cálculos mentais; estimular a concentração, raciocínio, perseverança e criatividade; promover a troca de idéias através de atividades em grupo; iniciar-se nos métodos de investigação científica; estimular a compreensão de regras, a percepção espacial, discriminação visual e a formação e fixação de conceitos.

Neste cenário, em que os jogos apresentam significativas contribuições ao processo de ensino e aprendizagem [Kishimoto 2010][Macedo 2009], cabe a realização de pesquisas que provenham aprimoramentos nas ferramentas didáticas no intuito de potencializá-las com novas funcionalidades capazes de melhorar o aproveitamento do tempo e dos recursos computacionais para o ensino, ampliando a disponibilidades de métodos e ferramentas para o campo educacional. A partir disso, busca-se munir o professor com recursos tecnológicos necessários para a exposição de seus conteúdos de forma diferenciada, com flexibilidade e rapidez ao aplicar as atividades aos alunos, para possibilitar o uso de aplicações em públicos heterogêneos, com diferentes capacidades e necessidades.

Aliado às pesquisas que envolvem a área de jogos destacam-se as influências provenientes da ciência cognitiva que têm se apresentado como importantes definidores da modelagem computacional na concepção de jogos digitais. Mais especificamente, a teoria da ergonomia cognitiva percebe o ser humano como um complexo sistema de processamento de informações [Heidrich 2007] e estuda os impactos do modo de apresentação, aprendizado, busca e transmissão destas informações. Diferente da Ergonomia clássica, a ergonomia cognitiva busca adaptar as Tecnologias de Informação e Comunicação (TICs) à mente humana, e não apenas às necessidades do corpo. Como um bom aprendizado depende da compreensão da mente e de como se aprende, as TICs precisam ser ergonomicamente bem concebidas, considerando as particularidades cognitivas.

Embora haja uma grande quantidade de ferramentas computacionais disponíveis para a educação atualmente, parte destes recursos não agrega qualidade ao processo de ensino, confundindo, desestimulando e dispersando a atenção dos educandos. Isso acontece, por exemplo, pelo uso de um design de tela complexo, que possui uma carga cognitiva elevada porque cada componente necessita ser percebido e interpretado pelo aprendiz, reduzindo sua capacidade para interpretação do conteúdo exposto.

Sweller (2011) dedica-se ao estudo da Teoria da Carga Cognitiva, conjunto universal de princípios que resultam em um ambiente de aprendizagem eficiente e que promovem um aumento na capacidade do processo de cognição humana. Estes princípios objetivam alinhar a interação humana com a tecnologia ao processo cognitivo, ou seja, disponibilizar um volume de informações compatível com a capacidade de compreensão humana.

Os jogos ganharam espaço no ambiente educacional, principalmente por permitir a participação ativa do aprendiz, experimentando e construindo conhecimento. Segundo Fletcher (2006), o uso de jogos educativos aprimora a capacidade de resolver problemas. O desenvolvimento de jogos alinhado com os princípios da teoria da carga cognitiva pode criar excelentes recursos tecnológicos, com significativa importância à evolução do processo de ensino e aprendizagem. 
Além disso, os estudos relacionados ao processo de cognição humana influenciaram fortemente o projeto de interface dos sistemas computacionais. Conhecer as necessidades e limitações dos usuários permitiu o desenvolvimento de interfaces mais amigáveis que agreguem valor na atividade de interação, garantindo maior qualidade no processo de ensino e aprendizagem, usando informações sobre as características de cada usuário e atendendo à sua afetividade, apoiado na teoria de que a satisfação do usuário o motiva e melhora a assimilação do conhecimento.

Oppermann (2009) separa o conceito de adaptação em sistemas computacionais em adaptatividade e adaptabilidade. Sistemas adaptativos podem modificar automaticamente suas próprias características, de acordo com a sua percepção das necessidades e características do usuário [Opperman 2009]. Segundo Ito (2011), as interfaces adaptativas se mostram como soluções para alguns dos problemas atuais na interação homem-computador. São necessárias interfaces capazes de se ajustar às necessidades do usuário. Já, sistemas adaptáveis disponibilizam ferramentas que permitem ao usuário alterar explicitamente certas características do sistema, a fim de adequá-lo ao seu afeto [Opperman 2009]. A literatura descreve a adaptação como uma solução para os problemas de quebra de fluxo conceitual ou narrativo, desorientação e sobrecarga cognitiva[De Bra 1998][Murray 2010].

Tais contribuições, advindas das áreas acima citadas, formam a base para a concepção do AdapterGame, que busca fornecer subsídios para que a informática seja utilizada de forma efetiva como suporte ao processo de ensino e de aprendizagem.

\subsection{Trabalhos Relacionados}

Alguns trabalhos desenvolvidos recentemente sejam em projetos no ambiente acadêmico ou produtos de caráter profissional, permeiam o tema abordado neste projeto. Dentre eles podem ser destacados: o Second Life, considerando o compartilhamento de conhecimento através de uma tecnologia baseada em jogos; o MyQuímica no sentido de objetivar a aplicação de jogos em ambientes educacionais para possibilitar, acelerar ou ampliar o domínio de um conteúdo exposto.

O Second Life caracteriza-se como um mundo virtual imersivo em 3D onde os usuários interagem com o mundo através de avatares, manipulando objetos e acessando conteúdos. Foi avaliado recentemente por algumas universidades como um ótimo ambiente para ensino e sob tal propósito foram criadas algumas universidades virtuais obtendo-se sucesso em seus objetivos. Já, o MyQuímica é um jogo desenvolvido para dispositivos móveis que propõe aos usuários vários desafios que consistem em arrastar compostos químicos da tabela periódica para um tubo de ensaio. O jogo permite visualizar informações sobre os elementos selecionados, dicas sobre o composto do desafio e informações complementares do composto. Também utiliza de artifícios de motivação, como o ranking ao final de cada jogo e a opção de pular para o desafio seguinte caso não tenha sucesso após várias tentativas.

Destes trabalhos, o Second Life apresenta como ponto negativo o fato de não ser um software livre, ou seja, para que uma instituição disponibilize conteúdos é necessária à locação de um espaço com custo mensal. Já, o MyQuímica apresenta de forma estática o conteúdo do jogo. 


\section{Visão Geral do AdapterGame}

O AdapterGame provê um conjunto de ferramentas para adaptação de jogos conforme conteúdo desejado. Para isso, apresenta uma estrutura modular com diferentes funcionalidades. O módulo "Gerenciador de Conteúdo", presente na estrutura, provê ferramentas que permitem a edição de conteúdos a serem inseridos no escopo dos jogos. Já, o módulo "Gerenciador de Jogos" provê um conjunto de jogos pré-definidos e disponibilizados pelo sistema para que sejam usados nas atividades de ensino propostas pelo usuário.

O módulo “Adaptador” é o responsável pela adequação da interface do jogo em relação ao conteúdo fornecido pelo usuário. Tanto os conteúdos como os jogos concebidos podem ser armazenados, possibilitando assim o reuso de conteúdos para concepção de novos jogos. A estrutura inicial proposta para o sistema AdapterGame é apresentada na Figura 1.

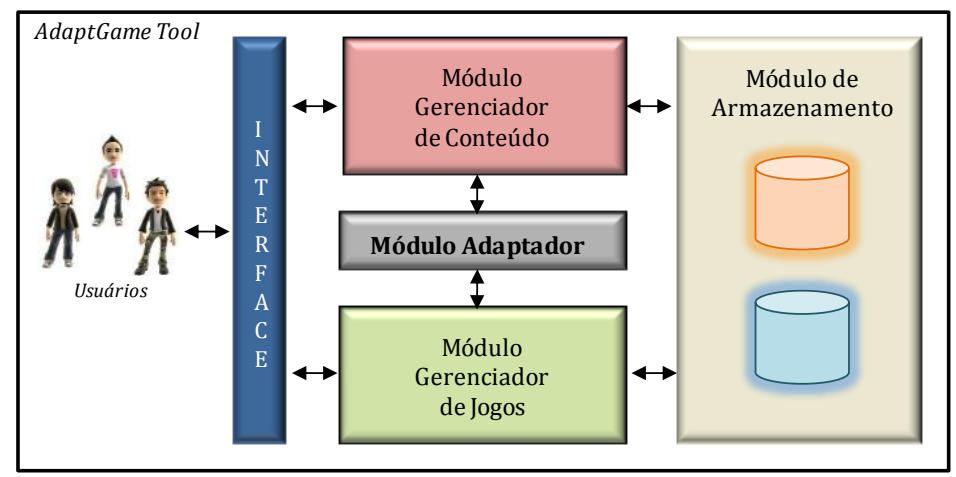

Figura1. Estrutura do Sistema AdapterGame

Considerando que a proposta parte do princípio que a carga cognitiva do usuário não deve ser sobrecarregada com informações que dificultam a interpretação da interface, prevê-se que a interação ocorra através de um pequeno conjunto de passos que apóiam o usuário na co-autoria de uma configuração para o jogo selecionado. $\mathrm{O}$ esquema apresentado na Figura 2 exemplifica as ações do usuário no escopo do sistema.

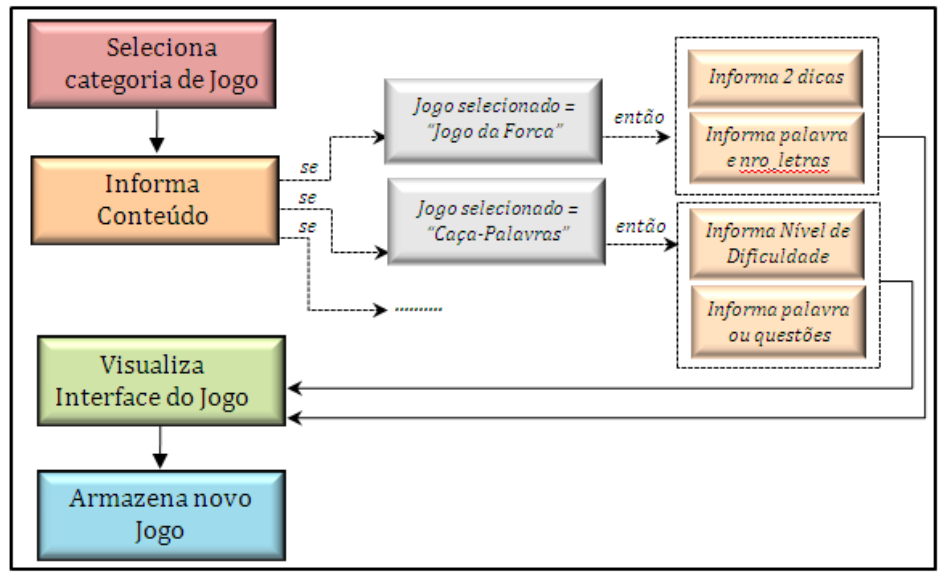

Figura 2. Principais atividades no escopo do AdapterGame

As atividades compreendem basicamente a seleção da categoria de jogo para a qual se deseja adaptar ao conteúdo, a inclusão do conteúdo através de interface própria, que varia para cada tipo de jogo selecionado e, após visualizar o jogo concebido, o 
usuário tem a opção de armazená-lo. A Figura 3 apresenta um protótipo de uma das ferramentas disponibilizadas pelo AdapterGame que permite a execução dos passos acima descritos.

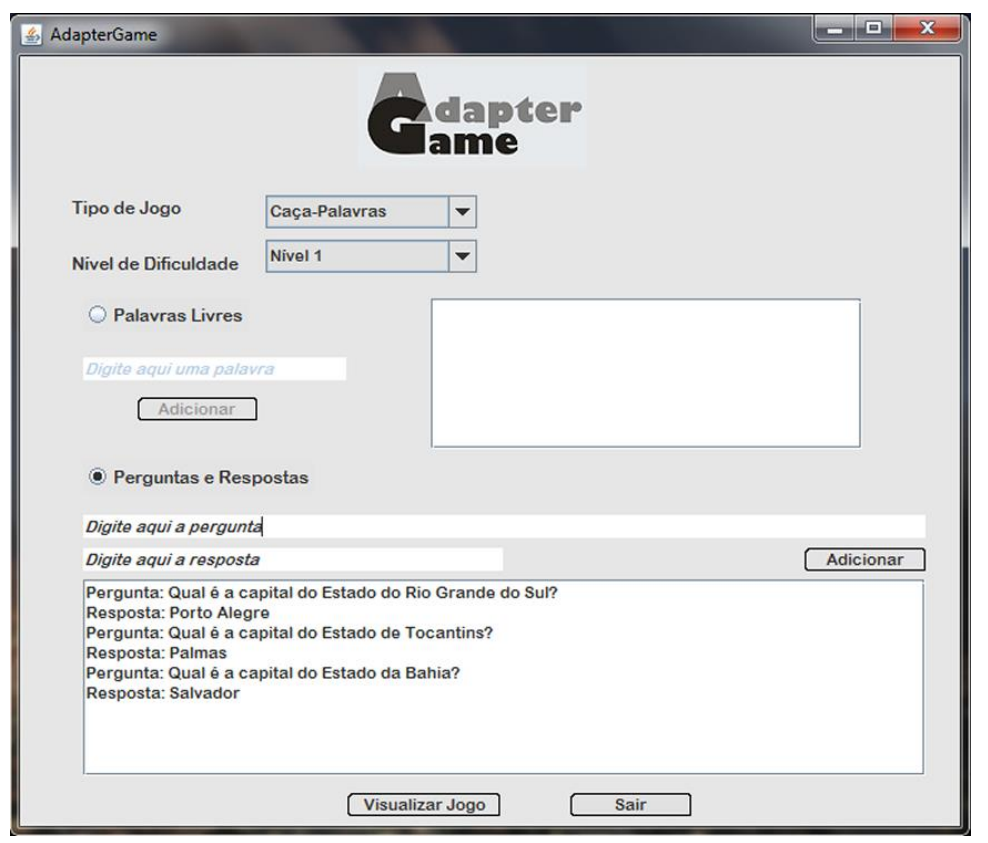

Figura 3. Protótipo da ferramenta para a concepção de jogos do tipo Caça-Palavras

A interface acima permite a seleção do tipo de jogo e a edição do conteúdo que será adicionado ao tipo de jogo selecionado. O item "nível de dificuldade" permite diferentes formas de disposição das palavras dentro do jogo, sendo que o nível 1 estrutura as palavras apenas na horizontal e vertical, o nível 2 inclui palavras também na diagonal e o nível 3 agrega todas as opções dos demais níveis e ainda adiciona palavras na ordem inversa. Uma vez gerado o novo jogo, o usuário tem a opção de armazená-lo ou não. Conforme pode ser observado, a interface é de fácil utilização e envolve a execução de poucos passos para o alcance dos objetivos. A Figura 4 apresenta o jogo Caça-Palavras já adaptado ao conteúdo inserido pelo usuário.

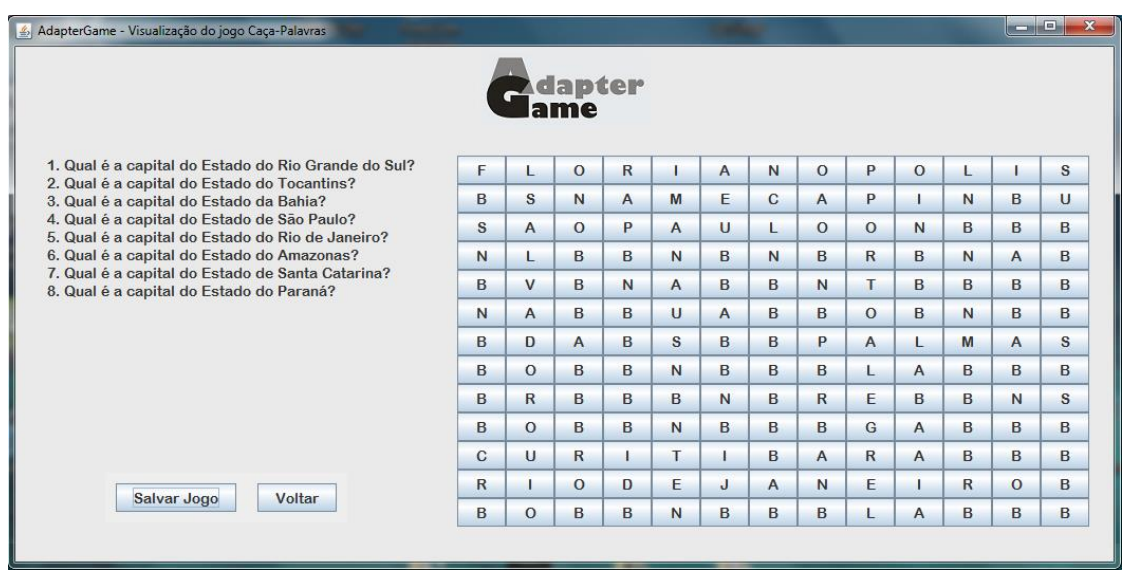

Figura 4. Interface do jogo Caça-Palavras com Conteúdo Adaptado

Para análise do impacto do AdapterGame foram realizados estudos de caso envolvendo sua aplicação em um ambiente real de sala de aula. Tais estudos permitiram que vários aspectos da proposta fossem avaliados, incluindo sua usabilidade, 
possibilidades de inclusão de conteúdos, pertinência dos tipos de jogos disponibilizados com as propostas pedagógicas para as quais se propõem, dentre outras. Uma descrição mais detalhada dos resultados obtidos a partir dos estudos de caso realizados é apresentada na próxima seção.

\section{Discussão e Resultados}

O AdapterGame foi projetado com vistas a estabelecer alternativas para que, a partir de um único ambiente, seja possível a concepção de jogos envolvendo conteúdos selecionados pelo professor e que sirva como apoio às suas práticas pedagógicas. Assim, o professor assume um papel fundamental e indispensável na avaliação do sistema.

Desta maneira, um estudo de caso foi realizado para permitir a realização de uma análise quanto ao impacto causado do sistema proposto. Para isso, contou-se com o envolvimento de um grupo de docentes composto por nove educadores, pertencentes a diferentes instituições de ensino e que atuam em diferentes disciplinas, dentre as quais Ciências, Português, Geografia, História e Espanhol. O processo avaliativo envolveu, em um primeiro momento, apenas educadores visto que pretende-se efetuar os ajustes necessários, identificados no processo, antes que o sistema seja avaliado por alunos. Assim, educadores foram incentivados a utilizar o sistema para produzir jogos envolvendo conteúdos específicos das disciplinas as quais ministram.

O uso do sistema por parte dos educadores promove as condições necessárias para que sejam realizadas avaliações envolvendo os fatores de qualidade estabelecidos por Vieira (2014) tais como, legibilidade (avalia a possibilidade de diferentes pessoas entenderem a aplicação); manipulabilidade(avalia a possibilidade de diferentes pessoas manipularem a aplicação com facilidade); utilizabilidade (avalia a conveniência e a viabilidade de sua utilização ao longo do tempo); validabilidade (avalia se a aplicação executa as funções para as quais foi projetada) e, fidedignidade (avalia a correspondência da aplicação com às necessidades identificadas). Os fatores de qualidade determinam a qualidade do ponto de vista dos usuários da aplicação. Para cada um dos fatores foram definidos critérios que comportam-se como atributos primitivos possíveis se serem avaliados. Tais critérios são apresentados na Tabela 1.

Tabela 1. Fatores de qualidade e critérios

\begin{tabular}{|c|l|}
\hline Fatores de Qualidade & \multicolumn{1}{c|}{ Critérios } \\
\hline \multirow{2}{*}{ Legibilidade } & $\begin{array}{l}\text { Coerência de apresentação do conteúdo } \\
\text { Clareza na proposta da aplicação } \\
\text { Interatividade com o usuário }\end{array}$ \\
\hline Manipulabilidade & $\begin{array}{l}\text { Grau de Compreensão sem a presença de um instrutor } \\
\text { Facilidade de uso } \\
\text { Possibilidade de uso do programa por iniciantes }\end{array}$ \\
\hline Utilizabilidade & $\begin{array}{l}\text { Oferecimento de diferentes caminhos para a solução de problemas } \\
\text { Possibilidade de adaptação conforme necessidade do usuário }\end{array}$ \\
\hline Validabilidade & $\begin{array}{l}\text { Apresentação fiel ao objetivo que propôs } \\
\text { Forma de avaliação das atividades desenvolvidas } \\
\text { Processamento rápido de dados, com eficiência e agilidade }\end{array}$ \\
\hline \multirow{2}{*}{ Fidedignidade } & $\begin{array}{l}\text { Favorecimento da utilização interdisciplinar } \\
\text { Potencialidade de utilização na sala de aula } \\
\text { Promoção da criatividade }\end{array}$ \\
\hline
\end{tabular}

Para identificar as percepções dos educadores quanto aos critérios avaliativos foram considerados valores numéricos de 1 a 10, coletados a partir de um instrumento 
elaborado para guiar o processo. As respostas de cada critério foram compiladas de acordo com o seu respectivo fator de qualidade e são apresentadas na Figura 5.

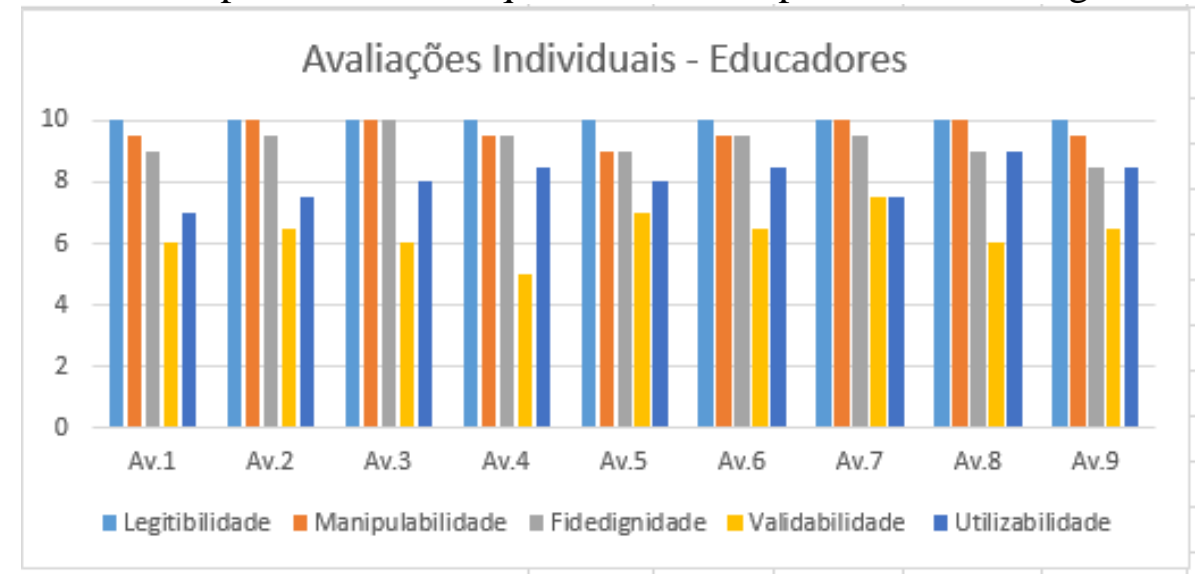

Figura 5. Dados da avaliação com educadores

Dos fatores considerados a "legitibilidade" e "manipulabilidade" obtiveram médias iguais a 10,0 e 9,67, respectivamente. Isto sugere que os avaliadores foram unânimes quanto ao entendimento do que é proposto pelo sistema e confirma a possibilidade do uso de forma simples, intuitiva e de fácil entendimento do sistema proposto. O fator "fidedignidade" obteve a média de 9,28 indicando uma avaliação favorável em relação à promoção da criatividade e ao potencial de uso do AdapterGame, de forma interdisciplinar, em sala de aula. Já, os fatores "utilizabilidade" e "validabilidade" obtiveram as médias 8,06 e 6,33, respectivamente. Quanto à utilizabilidade, todas as avaliações foram positivas no sentido de perceber a possibilidade de adaptação do conteúdo conforme a necessidade do usuário, no entanto, três avaliadores consideram que a ferramenta provê poucos recursos com vistas ao oferecimento de diferentes caminhos para a solução de problemas. Por fim, uma análise quanto às avaliações em relação ao fator "validabilidade" indica que a apresentação do AdapterGame está adequada ao objetivo que se propõem e que o processamento se dá de forma eficiente e ágil, mas, por outro lado, todos os avaliadores foram unânimes na percepção da ausência de instrumentos que permitam ao educador avaliar o desempenho dos aprendizes a partir das atividades propostas pelo ambiente.

Além da avaliação de tais fatores e critérios, também foram considerados os apontamentos dos avaliadores em relação às limitações do ambiente e sugestões de melhorias. Dentre os apontamentos que mais se destacaram incluem-se a necessidade de agregar ao AdapterGame um sistema de coleta de dados sobre a aprendizagem, a presença de mecanismos que estimulem a competitividade como forma de motivar os alunos e a possibilidade de personalização do ambiente de forma a prover recursos de acessibilidade. Assim, a partir da avaliação, novos estudos em relação ao modelo do AdapterGame têm sido realizados com vistas a incorporar os recursos/mecanismos sugeridos para a melhoria do ambiente. Tais estudos impulsionam a adição de regras de gamificação ao ambiente, bem como a personalização da interface como forma de prover recursos de acessibilidade. Além disso, há necessidade de prever um controle de acesso ao ambiente para prover as condições necessárias para a geração e armazenamento de informações a respeito do desempenho do aprendiz no processo de aprendizagem. Na Figura 6 são destacados os novos módulos incorporados no modelo do AdapterGame com vistas a atender as demandas identificadas. 


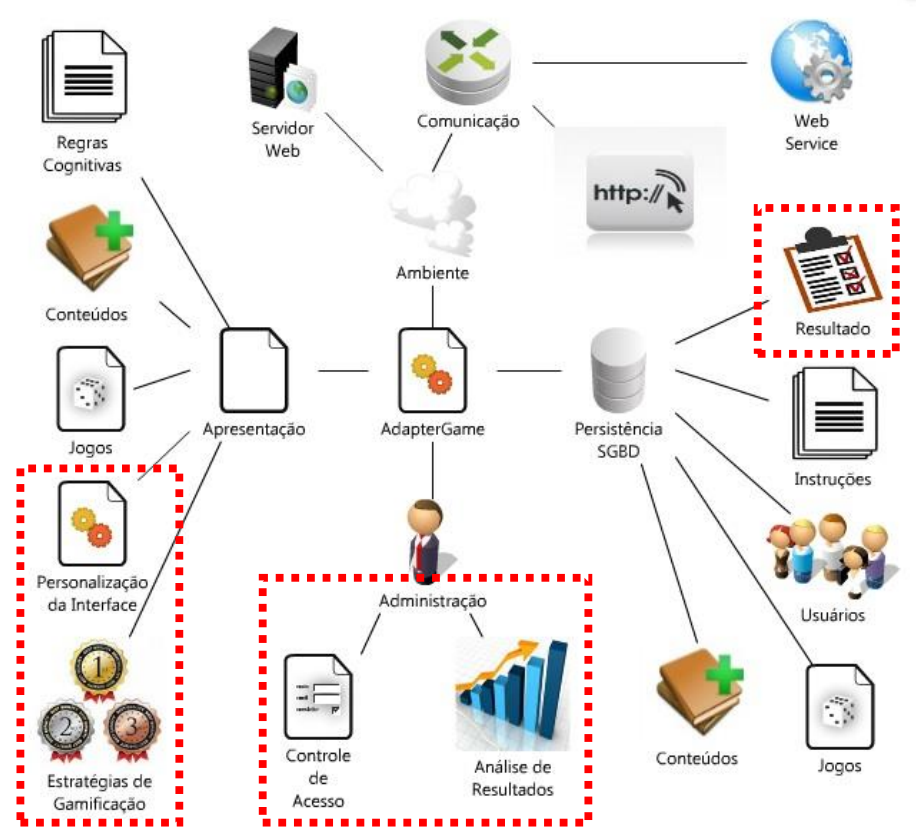

Figura 6. Modelo do AdapterGame estendido

Cabe destacar que o desenvolvimento dos novos recursos está em fase de estudos e implementações no ambiente. Após a inclusão das novas funcionalidades, o AdapterGame deverá ser avaliado novamente por educadores antes de ser disponibilizado para avaliação de aprendizes.

\section{Considerações Finais}

O ensino tem passado por grandes transformações ao longo dos anos, e, cada vez mais, existem esforços para tornar a aprendizagem mais atrativa e prazerosa. Neste movimento, insere-se a proposta do AdapterGame, que tem como ideia agregar conteúdo educativo de forma dinâmica em atividades lúdicas envolvendo o uso de jogos como o caça-palavra, palavras-cruzadas, jogo da forca, jogo da memória, dentre outros.

Com a concepção desta proposta vislumbram-se contribuições em diferentes contextos. Por um lado, no contexto computacional, por prover um modelo extensível promovendo subsídios para que outros profissionais da computação possam utilizá-lo e ampliá-lo para o desenvolvimento de jogos em outros contextos. Já, no contexto educacional, por possibilitar que educadores e educandos usufruam destes recursos no processo de ensino e aprendizagem de diferentes conteúdos, abordados em diversas disciplinas ao longo da sua formação.

Além disso, a proposta do sistema de jogos adaptáveis ao conteúdo considera diversas particularidades que o diferem da modelagem de software convencional incluindo inúmeros recursos que poderão contribuir significativamente se estendidos para diversos outros contextos, como por exemplo, a utilização em jogos sérios, desenvolvidos para treinamentos e simulações. Cabe ressaltar que a pesquisa e desenvolvimento do AdapterGame está em fase inicial, compreendendo ainda como tópicos de investigação/definição no escopo do sistema questões como o compartilhamento dos jogos dentro do ambiente, inclusão de estratégias de gamificação e incorporação de recursos de acessibilidade. Além disso, também é tópico de interesse na extensão do AdapterGame o uso de interação por gestos. 
Por fim, agradecimentos especiais a Secretaria de Ciência, Inovação e Desenvolvimento Tecnológico do Estado do Rio Grande do Sul que, por meio do Programa de Apoio à Polos Tecnológicos, apoia a realização das pesquisas relacionadas a este projeto.

\section{Referências Bibliográficas}

De Bra, P.; Calvi, L. AHA! An Open Adaptive Hypermedia Architecture. The New Review of Hypermedia and Multimedia. Taylor Graham Publishers, v. 4.1998

Dillenburg, D.J., Teixeira A.C. Uma proposta de avaliação qualitativa em ambientes virtuais de aprendizagem. In: Anais do Simpósio Brasileiro de Informática na Educação (SBIE), 22. Aracaju, 2011.

Fletcher, J. D., Tobias, S. Using computer games and simulations for instruction: A research review. Proceedings of the Society for Advanced Learning Technology Meeting, Orlando, FL February 2006.

Heidrich Regina de O., Medina; Güeba, Peirano; Salce, Fabrício André Peirano. Ergonomia Cognitiva e Usabilidade Aplicadas ao Design de Interface para Alfabetização. Anais do Workshop em Informática na Educação 2007. XVIII Simpósio Brasileiro de Informática na Educação - SBIE, 2007.

Hodgson, P.; Man, D.; Leung, J. "Managing the development of digital educational games". In: Proceedings of the 2010 Third IEEE International Conference on Digital Game and Intelligent Toy Enhanced Learning. Washington, DC, USA: IEEE Computer Society, 2010.

Ito, G. C.; Ferreira, M. G.; Sant'anna, Nilson. Uma ferramenta para geração de interfaces adaptativas. Instituto Nacional de Pesquisas Espaciais. ISBN: 972-8924 20-8. 2011

Kishimoto, Tizuka Morchida. (Org.) Jogo, Brinquedo, Brincadeira e a Educação. Ed. São Paulo: Cortez, 2010.

Macedo, Lino. Os Jogos e o Lúdico na Aprendizagem Escolar/Lino de Macedo, Ana Lúcia Sicoli Petty e Norimar Christe Passos, Porto Alegre: Artmed, 2009

Morsi, R.; Jackson, E. Playing and learning? Educational gaming for engineering education. In: Frontiers in Education Conference - Global Engineering: Knowledge Without Borders, Opportunities Without Passports, 2007.

Murray, T.; Shen, T.; Piemonte, J.; Condit, C.; Thibedeau, J. Adaptivity for Conceptual and Narrative Flow in Hyperbooks: The MetaLinks System. In: Adaptive Hypermedia and Adaptive Web-Based Systems: International Conference, AH 2000, Trento, Italy. Proceedings...Berlin: Springer, p. 155-166, 2010.

Opperman, Reinhard; Rashev, Rossen; Kinshunk. "Adaptability and Adaptivity in Learning Systems". Knowledge Transfer, v. 2, p.173-179, 2009.

Sweller, J. Evolution of human cognitive architecture. In B. Ross (Ed.). The Psychology of Learning and Motivation (Vol. 43, pp. 215-266). San Diego: Academic Press, 2011.

Vieira, Fábia Magali Santos. Avaliação de Software Educativo: Reflexões para uma Análise Criteriosa, 1999. Disponível em: www.edutec.net/edmagali2.htm. Acesso em: 11 de abril de 2014. 